\title{
HESITANCY PREVALENCE AND SOCIOCOGNITIVE BARRIERS TO CORONAVIRUS VACCINATIONS IN NIGERIA
}

\author{
ROTIMI OGUNTAYO ${ }^{1}$, ABAYOMI O. OLASENI ${ }^{1}$, ABIODUN EMMANUEL OGUNDIPE ${ }^{2}$ \\ ${ }^{1}$ Department of Psychology, Faculty of Social Sciences, University of Ilorin, Ilorin, Nigeria \\ ${ }^{2}$ Miami University Institute for the Environment and Sustainability (IES) Affiliate, Geography Department, \\ College of Arts and Sciences, USA
}

(C) 2021 Rotimi Oguntayo, Abayomi O. Olaseni, Abiodun Emmanuel Ogundipe

This is an open access article distributed under the Creative Commons Attribution-NonCommercial-NoDerivs license (http://creativecommons.org/licenses/by-nc-nd/3.0/)

DOI: $10.2478 /$ eras-2021-0008

\begin{abstract}
This study examined the prevalence, socioeconomic and cognitive barriers of coronavirus vaccinations in Nigeria. The study used an ex-post facto design. 526-participants were sampled using snowball sampling technique. A questionnaire pack containing socio-demographics and a 13-item adapted scale of SYKES was used. Findings revealed the prevalence of COVID-19 vaccination hesitancy (61.7\%). Adolescents (83.8\%) recorded more hesitancy than participants in early-adulthood (62.7\%), middle-adulthood $(53.1 \%)$, and late-adulthood (53\%). Males $(83.8 \%)$ showed hesitancy than the females $(33.3 \%)$. More so, the primary $(62.5 \%)$ and secondary school certificate holders $(41.4 \%)$ scored more on hesitancy than tertiary certificate holders $(36.0 \%)$. The identified perceived barriers to vaccinations are: safety $(91 \%)$; government distrust $(75.5 \%)$ and coerciveness $(65.7 \%)$, vaccines efficacy $(62.5 \%)$, complacency $(65.7 \%)$, and constraints to vaccination center $(55.5 \%)$. Conclusively, vaccination hesitancy was found more among males and younger respondents. Safety and efficacy of the vaccines, government distrust, coercive approach, and complacency were found as major barriers.
\end{abstract}

\section{Keywords}

cognitive barriers, socio-economic factors, COVID-19 vaccination, Nigeria, vaccines hesitancy

\section{Background}

The Coronavirus disease 2019 (COVID-19) pandemic has caused lasting wounds on the global world. Among these wounds are the scores of deaths and health-related challenges recorded during the first, second, and third waves in Nigeria. Consequently, many citizens are physically and emotionally affected (Agberotimi et al., 2020). Confirmed cases of the disease reached more than a 35 million by September 2021, and it has caused more than 1 million mortalities, particularly among the higher-risk population (WHO 2021a). More so, the pandemic has impacted Nigerians in significantly negative ways such as health, economic, familial, and workforce challenges (Nicola et al., 2020; Oguntayo et al., 2020).

To reduce the spread of the virus, strict preventive measures were imposed, such as hands hygiene, physical and social distancing, the compulsory use of face masks and gloves by the general public, and government imposition of stay-at-home orders at a point (Centers for Disease Control and Prevention 2021; World Health Organization (WHO) 2020a). Despite all these government efforts, a study from Nigeria has revealed that there is still low adherence to COVID-19 precautionary measures among citizens (Iorfa et al., 2020), while evidence abounds that the precautionary behavior is not strong enough to curtail the virus, the alternative mean of 
curtailing the virus was suggested to be a pharmacological method (vaccination) so that people could be immune to the disease (Iorfa et al., 2020; WHO 2020b).

Recently, the Centre for Disease Control reported that up to 63-million doses of the COVID-19 vaccine had been supplied to United State residents so far, and over 43.2 million doses of the vaccine have been received (Centers for Disease Control and Prevention 2021a). A country like the UK has vaccinated a total number of 12,844,193 individuals too (Centers for Disease Control and Prevention 2021b). Africa countries were not left out as the rollout of COVAX vaccines progresses; the first COVID-19 vaccination campaigns were done in Côte d'Ivoire and Ghana using COVAX doses (UK COVID-19 vaccination uptake plan 2021). So also, West Africa's largest country - Nigeria - has got its first batch of coronavirus vaccines, to make the third country to receive the vaccines in Africa and receive the shots through COVAX (UNICEF, 2021). Other African nations notwithstanding are awaiting the distributing agencies to dispatch theirs (Aljazeera, February $3^{\text {rd }}, 2021$ ).

Despite that the COVID-19 pandemic's risk has exacerbated the awareness and the need for an effective vaccine (Iorfa et al., 2020), the vast majority of people may still not accept the vaccines, for instance, a recent study revealed that newer vaccines bring about an increase in hesitancy (WHO 2021b). Another survey conducted in the United States reported that when COVID-19 vaccines are available to the public, up to $49 \%$ of the respondents accept to receive it, while $31 \%$ of them were indifferent, and $20 \%$ displayed signs of hesitancy (Brunson \& Schoch-Spana, 2020). These referred studies, together with the fact that a great number of people are seeking alternative vaccination timelines or hesitating vaccination as a whole, have provided evidence that COVID-19 vaccine hesitancy has turned into a serious concern that needed to be addressed globally (AP-NORC poll, 2021). Consequently, one could conclude that vaccine resistance represents a global threat to health, that can seriously destroy the implementation and achievement expected, such as public acceptance of coronavirus vaccines.

Past studies have revealed that more people disparage vaccination generally (Blume, 2006). For instance, people are skeptical about some vaccines and sometimes, because of that, hesitate to accept vaccination either for their children, relatives, or self (Dubé et al., 2013). Also, some citizens are afraid of the reactions or side effects of a vaccine, and some citizens have the view that vaccine-preventable ailments are not serious, they lack trust in government and have no belief in herd immunity (Benin et al., 2006; Blume 2006; Corey et al., 2020; UNICEF, 2021). Meanwhile, government trust has been found to influence vaccination acceptance, vaccination effectiveness, healthcare experts, and healthcare delivery (Centers for Disease Control and Prevention, 2012b).

Despite the negative dispositions of the public towards vaccines, it has been proven as a successful measure of disease prevention for many years (WHO, 2020c). Vaccine refusal and hesitancy are a globally concern especially during the COVID-19 pandemic (WHO, 2020c). This makes WHO declare vaccine refusal and hesitancy in 2019 as one of the top 10 threats to health (WHOb, 2020). The determinants of vaccine unacceptance, as found in diverse studies, include conspiracy theories, religious beliefs, infodemic, individual beliefs, and anxiety of vaccine safety as a result of myths (Al-Qerem \& Jarab, 2021; Iorfa et al., 2020).

According to Al-Qerem et al. (2021), associations exist between vaccines, beliefs, economics, and social values of any society. Unfortunately, in Nigeria no sufficient studies have been conducted to assess the Nigerian population's dispositions to COVID-19 vaccination. To the best of our knowledge, no past published study has examined the intent of Nigerian citizens to be vaccinated against COVID-19, nor a study to evaluate the current vaccine acceptability and possible socio-cognitive barriers to the vaccines' acceptance after its availability to the citizens. Therefore, this study is timely to create evidence-based policies that will guide the government and non-governmental organizations on how to tackle vaccine hesitancy (UNICEF, 
2021). The objective of this study is to investigate the prevalence of the COVID-19 vaccines hesitancy and acceptance, socio-economic and cognitive barriers to vaccine acceptance among some selected Nigerians.

\section{Data and Methods}

\section{Design}

This study adopted a cross-sectional blend of ex-post-facto design, which allowed effortlessly the engagement of the Nigerian population across stratum of social classifications to report barriers or dispositional factors to acceptance of vaccinations during delta wave declaration in Nigeria. This survey is necessary to implement appropriate policy plans to respond appropriately to hesitancy determinants that are peculiar to Nigerians.

\section{Setting and Participants}

The study was conducted evenly across the six geopolitical zones of the country. Nigeria is a large, densely populated West Africa Country, segmented into six geo-political zones and two regions (the Southern and Northern Nigeria). The country was assumed to have over 196 million population as of 2018. According to World Health Organization, the country had reported over 200.884 cases, 206 newly reported incidents, and 2.128 confirmed deaths due to coronavirus infections. The general population of the study covered participants aged 18 years and above among the 36 states in Nigeria, as well as the Federal Capital Territory of the country.

Participants of this study were five hundred and twenty-six $(n=526)$ Nigerians $(58.5 \%$ males and $41.6 \%$ females), with diverse dispositions to acceptance of COVID-19 vaccination.

\section{Instruments}

The questionnaire was made up of two sections: Section A and B. Section A was comprised of socio-demographics of respondents' data, such as age, gender, marital status, highest educational attainment, state of residence, occupation, religion among others. Section $\mathrm{B}$ was an open-ended questionnaire meant to identify the socio-economic and cognitive barriers to acceptance of COVID-19 vaccinations. This assessment tool is a 13-item with a Likert-type scale, adapted from SYKES United States Survey (2020); it assesses vaccine acceptance (items 1 and 2), acceptance to participate in clinical trials (items 3 and 4), risk perception of COVID19 vaccine (items 5, 6, 7, 8 and 9), and other local content of socioeconomic factors that could moderate the reception of the vaccines (items 10,11, 12 and 13). Items were reworded to make them fit for the aims of this study (for example: "Now that government of Nigeria is to provide free COVID-19 vaccine, would you accept to be vaccinated?", and "Are you worried about accepting the Coronavirus vaccination injection?"), acceptance to participate in clinical trials (e.g., "If approached, would you accept to participate in COVID-19 vaccine trial?"), risk perception of COVID-19 vaccine (e.g., "I worry about the vaccine that it could cause another health complications"), other local content psychosocial moderators (e.g., "If an employer makes the reception of vaccine prerequisite to retaining or gaining a job, would receive the vaccine?"), etc. Participants rated these items on a 3-point Likert-type scale (yes=2, Don't know $=1$, and no $=0$.). A reliability coefficient (Cronbach's alpha) of 0.71 was obtained during the pilot testing of the scale, while the current data set yielded 0.75 . 


\section{Procedures and Sampling Technique}

Respondents were invited via social media (WhatsApp and Facebook posts) to complete an online survey on the socio-economic and cognitive barriers to the acceptance of COVID-19 vaccination during delta variant declaration, using Google form website and printed questionnaires. Participants were recruited online through a snowball sampling technique with a consent form attached to it. The snowball technique was considered appropriate due to the partial social restrictions enforced during the study in Nigeria. Some individual adults on researchers' social media contacts across geo-political zones, States, local governments, cities, and towns in Nigeria have shared the Google link. Prospective participants were then driven to share the link with other residents in their contacts in other parts of the country. Citizens with access to the internet, who can read and understand the English Language, and were ready to sign the informed consent, were the only ones included in this study. This helped in the collection of five hundred and twenty-six $(n=526)$ responses through the Google link.

Ethical review and approval were not made compulsory for the study on human participants following the local legislation and institutional requirements. However, written informed consent was implied via the completion of the questionnaire/survey. Other ethical considerations, such as participants' confidentiality and autonomy, were strictly observed with informed consent embedded in the questionnaire.

\section{Statistical Analyses}

Data were properly checked and subjected to statistical analysis using the Statistical Package for the Social Sciences (SPSS 20). Descriptive statistics and inferential statistical (chisquare) were used to analyze the formulated objectives.

\section{Results}

Objective 1: Prevalence of vaccine acceptance and hesitancy in Nigeria

\section{Graph 1 Showing the Prevalence of Acceptance-Hesitancy of COVID-19 Vaccination among Nigerians}

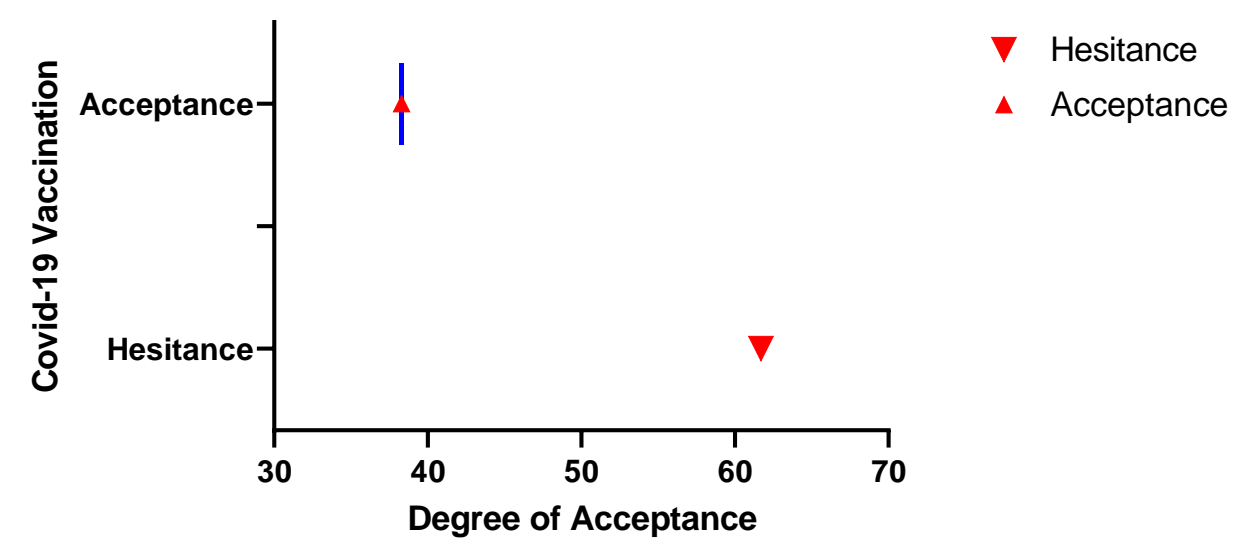

Graph 1 above revealed that $61.7 \%$ of the study participants show hesitancy towards being vaccinated in Nigeria, while $38.3 \%$ exhibit acceptance to COVID-19 vaccination in Nigeria. 
Objective 2: Justify the socio-demographic (age categories, sex, marital status, education qualification, locality of settlement) factors influencing hesitancy of COVID-19 vaccine

\begin{tabular}{|c|c|c|c|c|c|c|}
\hline \multicolumn{7}{|c|}{$\begin{array}{l}\text { Table } 1 \text { showing the influence of socioeconomic status c } \\
\text { Nigerians }\end{array}$} \\
\hline Socio-Economics & \multicolumn{2}{|c|}{ COVID-19 Vaccination } & \multirow{2}{*}{$95 \% \mathrm{CI}$} & \multirow{2}{*}{$\mathrm{df}$} & \multirow{2}{*}{$\chi^{2}$} & \multirow{2}{*}{$\eta_{p}^{2}$} \\
\hline Age & Acceptance (\%) & Hesitance (\%) & & & & \\
\hline Adolescents & 16.2 & 83.8 & $0.014-0.020$ & \multirow{4}{*}{3} & \multirow{4}{*}{$10.09 * *$} & \multirow{4}{*}{0.138} \\
\hline Early Adults & 62.7 & 37.3 & $0.015-0.022$ & & & \\
\hline Middle Adults & 46.9 & 53.1 & $0.014-0.021$ & & & \\
\hline Late Adults & 53.0 & 47.0 & $0.028-0.037$ & & & \\
\hline \multicolumn{7}{|l|}{ Gender } \\
\hline Male & 49.5 & 50.5 & $0.00-0.000$ & \multirow{2}{*}{1} & \multirow{2}{*}{$15.32 * *$} & \multirow{2}{*}{0.171} \\
\hline Female & 66.7 & 33.3 & $0.00-0.000$ & & & \\
\hline \multicolumn{7}{|l|}{ Education } \\
\hline Primary & 37.5 & 62.5 & $0.170-0.192$ & \multirow{3}{*}{2} & \multirow{3}{*}{$07.89^{* *}$} & \multirow{3}{*}{0.122} \\
\hline Secondary & 58.6 & 41.4 & $0.205-0.290$ & & & \\
\hline Tertiary & 64.0 & 36.0 & $0.161-0.181$ & & & \\
\hline \multicolumn{7}{|l|}{ Marital Status } \\
\hline Married & 57.5 & 42.5 & $0.157-0.176$ & \multirow{3}{*}{2} & \multirow{3}{*}{05.12} & \multirow{3}{*}{ NA } \\
\hline Unmarried & 55.4 & 45.6 & $0.172-0.192$ & & & \\
\hline Separated & 16.7 & 83.3 & $0.285-0.309$ & & & \\
\hline \multicolumn{7}{|l|}{ Location } \\
\hline Rural & 56.7 & 43.3 & $0.12-0.139$ & \multirow{2}{*}{1} & \multirow{2}{*}{.001} & \multirow{2}{*}{ NA } \\
\hline Urban & 56.6 & 43.4 & $0.28-0.029$ & & & \\
\hline
\end{tabular}

Table 1 showing the socio-economic relationship between socio-economic status and COVID-19 vaccination revealed a significant association between participants' age and COVID-19 vaccination, such that the $13.8 \%$ variance observed in the decision towards vaccination was accounted for by respondents' age categories $\left(\chi^{2}=10.09 ; \mathrm{p}<.01 ; \eta \mathrm{p} 2=\right.$ $0.138)$. It was further revealed that respondents in adolescent (83.8\%) and middle adulthood (53.1\%) classifications exhibited hesitancy towards vaccination, while the majority of the respondents in early adulthoods $(62.7 \%)$ and late adulthoods $(53 \%)$ exhibited acceptance towards vaccination.

It was further found that there is a significant association between participants' sex and COVID-19 vaccination, such that the $17.1 \%$ variance observed in the decision towards vaccination was accounted for by respondents' affiliated $\operatorname{sex}(\chi 2=15.32 ; \mathrm{p}<.01 ; \eta \mathrm{p} 2=0.171)$. It was further revealed that male respondents $(83.8 \%)$ show more hesitancy towards vaccination than their female counterparts $(33.3 \%)$. 
The study further revealed a significant association between participants' level of education and COVID-19 vaccination, such that the $12.2 \%$ variance observed in the decision towards vaccination was accounted for by respondents' academic qualification $(\chi 2=07.89 ; \mathrm{p}$ $<.01 ; \eta p 2=0.122)$. It was further revealed that primary school certificates holders $(62.5 \%)$ show more hesitancy towards COVID-19 vaccination compared to counterparts with secondary (41.4\%) and tertiary education qualifications (36.0\%).

However, on the contrary, it was revealed that marital status $(\chi 2=05.12 ; \mathrm{p}>.05)$ and respondents' location $\left(\chi^{2}=0.001 ; \mathrm{p}>.05\right)$ are not significantly associated with COVID-19 vaccination.

Objective 3: Examine the prevailing analyses of cognitive barriers towards hesitancy behavior to the COVID-19 vaccine.

\section{Chart 2 showing the Prevailing Cognitive Barriers to COVID-19 Vaccination}

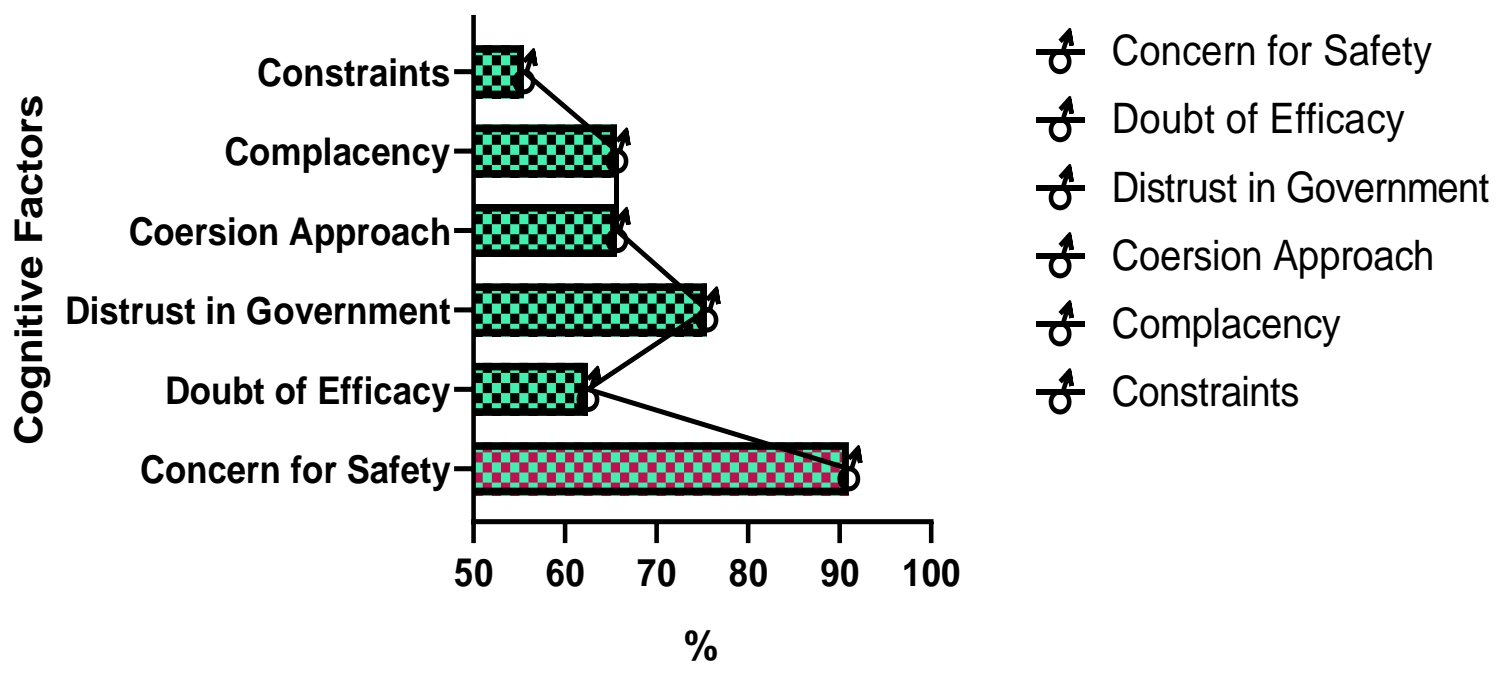

Chart 2 above revealed that the majority of the participants identified concern for safety $(91 \%)$ as the cognitive reason for hesitating to accept COVID-19 vaccination. Other implicated factors are distrust in government $(75.5 \%)$, doubt of efficacy $(62.5 \%)$, coercive approach $(65.7 \%)$, complacency $(65.7 \%)$, and constraints or convenience to centers $(55.5 \%)$.

\section{Discussion}

The present study examined the socio-economic determinants and cognitive barriers towards coronavirus vaccination among Nigerians. Findings revealed that a high number of the participants showed hesitancy towards being vaccinated in Nigeria. The results revealed $61.7 \%$ of the participants' hesitancy towards being vaccinated in Nigeria. This is similar to data obtained in certain countries; for instance, UK, Kuwait, Saudi Arabia, Russia, and Italy (Abedin et al., 2021; WHO, 2020c; Danchin et al., 2020). However, some studies contradicted our results such as studies conducted in China, Malaysia, Indonesia, French, Ecuador, and Brazil (Abedin et al., 2021; Sarasty et al., 2020; Wang et al., 2020; Ward et al., 2020; WHO, 2020c). In sub-Saharan Africa, the COVID-19 is a novel disease, as a result the observed mistrust and 
distorted beliefs about the vaccine, as well as the distrust of government, and information gaps may be reasons for results obtained in the current study.

There was a significant association between participants' age and COVID-19 vaccination, such that, the respondents in adolescence and middle adulthood classifications exhibited hesitancy towards vaccination, while the majority of the respondents in early adulthoods and late adulthoods exhibited acceptance towards vaccination. More so, results showed that there is a significant association between participants' sex and COVID-19 vaccination, such that, the male respondents showed more hesitancy towards vaccination than the female counterparts. So also, the participant's level of education and COVID-19 vaccination was associated; participants with primary school certificates showed more hesitancy towards COVID-19 vaccination compared to counterparts with secondary and tertiary education qualifications. This is in line with a study conducted in Bangladesh - it was found that there was an association between gender (male gender), age, and lower educational status, and coronavirus vaccine acceptance (Abedin et al., 2021). Precisely, the current study showed that the male gender was more likely to hesitate to be vaccinated and as the age of participants decreases so also the tendency for vaccine hesitancy. Acceptability by age in our study was recorded among the participants between ages 51-60 years, and low education was a positive factor in vaccine hesitancy, which is inconsonant with some other previous studies (Malik et al., 2021; Nolna et al., 2018). On the contrary, it was revealed that marital status and respondents' location were not significantly associated with COVID-19 vaccination acceptance. However, the current result contradicted a past study (Abedin et al., 2021). This contradiction could be that both participants (in a relationship or not, married or not, in rural or urban locations) have adequate or equal awareness of coronavirus. Also, maybe their sources of information about the coronavirus vaccine are equal, which initiates a similar disposition towards the vaccines. This indicates a need to focus all populations across different geographical regions, and marital statuses.

The majority of the participants identified concern for safety as the highest cognitive reason for hesitating to accept COVID-19 vaccination. This is similar to past results obtained from other countries (Malik et al., 2021; McKee et al., 2018; Ward, 2020; Wang et al., 2020). In this study the biggest concern of the participants was the safety of the vaccine. Some other sample characteristics have significant implications among the participants' cognitive barriers to be vaccinated, such as distrust in government, doubt of the efficacy of the vaccine, resistance to the coercive approach of authority, complacency, and constraints or convenience to centers. From the past studies, the efficacy of a new vaccine is one of the most public concerns (Determann et al., 2014; Kreps et al., 2020), distrust of government, resistance to the coercive approach of authority and doubt of the efficacy of a new vaccine (Al-Qerem, 2020; Mckee et al., 2018; WHO, 2021a). This concern could be curbed once the vaccine's adequate evidence of safety is made available through empirical results or published data that would show that the vaccine is reliable and not harmful. Also, lower vaccination intention among male participants was observed in some studies (Wang et al., 2020; WHO, 2020c). The recognition of these factors could probably serve as the limelight to more awareness campaigns directed to these affected populations to increase the vaccination acceptance rate generally.

\section{Strengths and limitations of this study}

This study comprehensively assessed the socioeconomic and cognitive barriers of coronavirus vaccine acceptance in Nigeria. The prevalence of vaccine hesitancy was established among the public. Also, the socio-economic determinants of the hesitancy were revealed and the cognitive barriers such as government mistrust, misinformation on vaccine safety, perceived imposition from the government, and doubt on the effectiveness of the vaccines were found as 
determinants of the vaccine hesitancy in this study. All these served as strengths of this study. However, this is one of the first studies in Nigeria that has investigated the prevalence of vaccine acceptance considering the socioeconomic and cognitive barriers. To the authors' best of knowledge, it is also the first study to consider implicit factors that could militate against the acceptance of the vaccine globally using an online survey method to collect data. Despite these relevancies, the study needed to be interpreted with care because of these limitations; most internet illiterate citizens were denied participation in this study, and a study like this is prone to selection and responses biases.

\section{Conclusion and recommendations}

The present study examined the socio-economic determinants and cognitive barriers towards coronavirus vaccinations among Nigerians. The study concluded that a high number of Nigerians displayed hesitancy towards being vaccinated. Age and COVID-19 vaccination are associated with citizens in late adolescence and middle adulthood classifications, who exhibited more hesitancy towards vaccination, while the majority of the respondents in early adulthoods and late adulthoods exhibited acceptance towards vaccination. More so, the sex of the citizens and COVID-19 vaccination is related; more hesitancy towards vaccination was found in the males compared to the female counterparts. So also, the participant's level of education and COVID-19 vaccination was associated; participants with primary school certificates showed more hesitancy towards COVID-19 vaccination compared to counterparts with secondary and tertiary education qualifications counterparts. However, on the contrary, it was revealed that marital status and respondents' location were not significantly associated with COVID-19 vaccination. In addition, the majority of the participants identified concern for safety as the highest cognitive reason for hesitating to accept COVID-19 vaccination. Other implicated factors are distrust in government, doubt of efficacy, resistance to coercive approach, complacency, and constraints or convenience to centers. Therefore, the study recommends thus:

1. Media and communication experts should structure their message targeting the right audiences effectively, especially the male and young citizens.

2. The government at all levels should take effective measures to develop a focus-based vaccine campaign strategy for young people, especially male citizens. Health promotion officers should take misinformation of coronavirus vaccine among the public generally as a matter of urgency. Especially mistrust of government, the safety of the vaccine, and reinforcement of vaccine acceptance instead of using force against the citizens. This message should be designed in a way that the public will easily understand and be motivated to accept the vaccines; thereby, grow trust in the government and healthcare system.

3. The government and its health agencies should also collaborate with the association of psychologists in Nigeria to get expert-structured-communication channels and be guided on the use of behavioral science methods of presenting or passing messages to the public with less forceful, and non-anxiety provoking manners, but in motivating manners for citizens to gain insight to a positive health care accessibility that will yield convincing and positive feedback on coronavirus vaccines.

\section{REFERENCES}

Abedin, Minhazul et al. (2021). Willingness to vaccinate against COVID-19 among Bangladeshi adults: Understanding the strategies to optimize vaccination coverage. PLOS ONE 16(4): e0250495.

Agberotimi, Samson F et al. (2020). Interactions Between Socioeconomic Status and Mental Health Outcomes in the Nigerian Context Amid COVID-19 Pandemic: A Comparative Study. Frontiers in Psychology 11: 559819. 
Rotimi Oguntayo, Abayomi O. Olaseni, Abiodun Emmanuel Ogundipe, Hesitancy Prevalence and Sociocognitive Barriers to Corona-Virus Vaccinations in Nigeria

DOI: $10.2478 /$ eras-2021-0008

Al-Qerem, Walid A., and Jarab Anan S. (2021). COVID-19 Vaccination Acceptance and its Associated Factors Among a Middle Eastern Population. Frontiers in Public Health 9: 632914.

AP-NORC poll. (2021). Expectations for a COVID-19 Vaccine. Retrieved September 9, 2021 (https://apnorc.org/projects/expectations-for-a-COVID-19-vaccine/)

Blume, Stuart. (2006). Anti-vaccination movements and their interpretations. Social Science and Medicine 62(3): 628-642.

Benin, Andrea L et al. (2006). Qualitative analysis of mothers' decision-making about vaccines for infants: the importance of trust. Pediatrics 117, 5: 1532-1541.

BBC News June 8 (2021). COVID-19 vaccines: Why some African states can't use their vaccines. Retrieved July 22, 2021 (https://www.bbc.com/news/56940657).

Brunson, Emily K, and Schoch-Spana Monica. 2020. A social and behavioral research agenda to facilitate COVID19 vaccine uptake in the United States. Health security 18: 338-344.

Centers for Disease Control and Prevention. (2021a). How to protect yourself and others. Retrieved September 22, 2021 (https://www.cdc.gov/coronavirus/2019-ncov/prevent-getting-sick/prevention.html).

Centers for Disease Control and Prevention. (2021b). Reporting COVID-19 vaccination in the United States. Retrieved September 22, 2021 (https://www.cdc.gov/coronavirus/2019-ncov/vaccines/reportingvaccinations.html).

Corey, Lawrence et al. (2020). A strategic approach to COVID-19 vaccine RandD. Science 368: 948-950.

Danchin, Margie et al. (2020). Preparing the public for COVID-19 vaccines: how can general practitioners build vaccine confidence and optimise uptake for themselves and their patients? Australian Journal of General Practice 49: 625-9. doi: 10.31128/AJGP-08-20-5559

Dubé, Eve et al. (2013). Vaccine hesitancy: An overview. Human Vaccines and Immunotherapy 9(8): 1763-73. doi: $10.4161 / \mathrm{hv} .24657$

Iorfa, Steve K et al- (2020). COVID-19 Knowledge, Risk Perception, and Precautionary Behavior Among Nigerians: A Moderated Mediation Approach. Frontiers in Psychology 11: 566773. doi: 10.3389/fpsyg.2020.566773

Jordan Times. (2020). Jordan to begin COVID-19 vaccination drive by February-Health minister. Available online at: https://www.jordantimes.com/news/local/jordan-begin-COVID-19-vaccination-drivefebruaryhealth-minister (accessed January 3, 2021).

Malik, Asmara et al. (2021). Acceptance of COVID-19 vaccine in Pakistan among health care workers. PLoS ONE 16(9): e0257237. https://doi.org/10.1371/journal.pone.0257237

McKee, Chephra, and Bohannon Kristin. (2018). Exploring the reasons behind parental refusal of vaccines. Journal of Pediatric and Pharmacological Therapy 21: 104-9. doi: 10.5863/1551-6776-21.2.104

Nicola, Maria et al. (2020). The socio-economic implications of the coronavirus pandemic (COVID-19): a review. International Journal of Surgery 78: 185-93.

Nolna, Sylvie K et al. (2018). Factors influencing the performance of routine immunization in urban areas: A comparative case study of two cities in Cameroon Douala and Yaoundé. Vaccine 36: 7549. https://doi.org/10.1016/j.vaccine.2018.10.048.

Oguntayo, Rotimi et al. (2020). Spousal Violence in The Era of COVID-19 Lockdown: The Implication of Socioeconomic Distress and Contextual Factors. Ilorin Journal of Economic Policy 7(3): 51-60. Available at: https://ideas.repec.org/a/ris/ilojep/0036.html\#download

Sarasty, Oscar et al. (2020). The demand for a COVID-19 vaccine in Ecuador. Vaccine 38: 8090-8.

Snyder, Christopher M et al. (2020). Designing Pull Funding. For A COVID-19 Vaccine. Health Affairs 39(9): 1633-1642

SYKES U.S Survey. (2020). SURVEY REPORT: American Perceptions of COVID-19 Vaccines. Retrieved May 17, 2021 (https://www.sykes.com/resources/reports/2020-covid19-vaccine-survey-report/)

UK COVID-19 vaccination uptake plan. (2021). Vaccination for coronavirus. Retrieved September 23, 2021 (https://www.gov.uk/government/publications/COVID-19-vaccination-uptake-plan)

UNICEF. (2021). First COVID-19 COVAX vaccine doses administered in Africa. September 23, 2021(https://www.unicef.org/press-releases/first-COVID-19-covax-vaccine-doses-administered-africa)

Wang, Jiahao et al. (2020). Acceptance of COVID-19 Vaccination during the COVID-19 Pandemic in China. Vaccines 8: 482. doi: 10.3390/VACCINES8030482

Ward, Jeremy K et al. (2020). The French public's attitudes to a future COVID-19 vaccine: the politicization of a public health issue. Social Science Medical 265: 113414. doi: 10.31235/osf.io/xphe9

World Health Organization. (2021a). COVAX announces additional deals to access promising COVID-19 vaccine candidates; plans global rollout starting Q1 2021. September 23, 2021 (https://www.who.int/emergencies/diseases/novel-coronavirus-2019/interactivetimeline?gclid=CjwKCAjw2bmLBhBREiwAZ6ugo58RjB3aKRO_e5p_unPSMu5beCjAeljJrhSi9SIyW E7dQzjM7tmnYxoCAAkQAvD_BwE\#event-115) 
World Health Organization Regional Office for Africa. (2021b). COVAX expects to start sending millions of COVID-19 vaccines to Africa in February. September 23, 2021 (https://www.afro.who.int/news/covaxexpects-start-sending-millions-COVID-19-vaccines-africa-february)

World Health Organization. (2020a). Coronavirus disease (COVID-19): Herd immunity, lockdowns and COVID19. September 23, 2021 (https://www.who.int/news-room/q-a-detail/herd-immunity-lockdowns-andcovid-19?gclid=CjwKCAjw2bmLBhBREiwAZ6ugozLc2rzVmE0xvMU9pVKucI9VCCNi-i6gD78YYr_f_Maw6aO9TBFHBoCjS8QAvD_BwE\#)

World Health Organization [WHO]. (2020b). Responding to Community Spread of COVID-19. Geneva: World Health Organization. September 23, 2021 (https://www.who.int/publications/i/item/responding-tocommunity-spread-of-covid-19).

World Health Organization. (2020c). Behavioral Considerations for Acceptance and Uptake of COVID-19 Vaccines: WHO Technical Advisory Group on Behavioral Insights and Sciences for Health, Meeting Report, 15 October 2020. World Health Organization. September 23, 2021 (https://apps.who.int/iris/handle/10665/337335). 